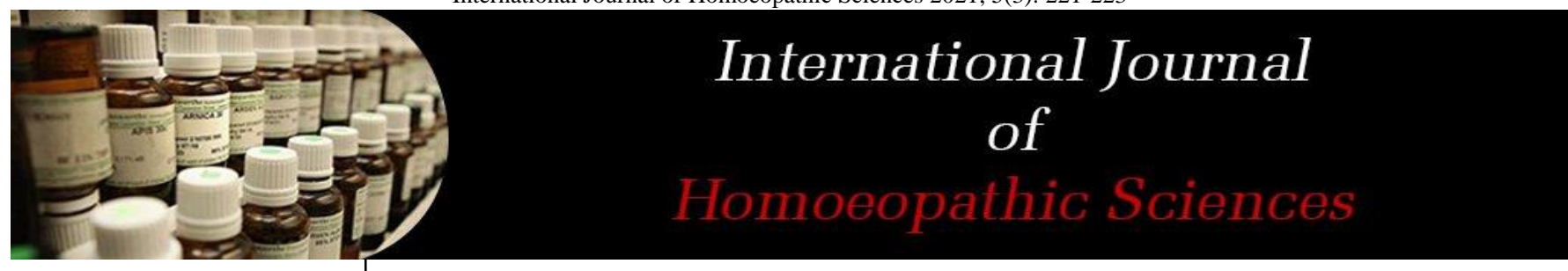

E-ISSN: 2616-4493 P-ISSN: 2616-4485 www.homoeopathicjournal.com IJHS 2021; 5(3): 221-223

Received: 21-05-2021

Accepted: 27-06-2021

Dr. Lakshmi GL

Associate Professor,

Department of Practice of

Medicine Govt Homoeopathic

Medical College Trivandrum,

Kerala, India
Corresponding Author:

Dr. Lakshmi GL

Associate Professor,

Department of Practice of

Medicine Govt Homoeopathic

Medical College Trivandrum,

Kerala, India

\title{
Persistent cough after COVID-19 Pathophysiology and homoeopathic management
}

\section{Dr. Lakshmi GL}

DOI: https://doi.org/10.33545/26164485.2021.v5.i3d.426

\begin{abstract}
COVID-19 is caused by severe acute respiratory syndrome coronavirus2 (SARS-CoV-2), which infects host cells via angiotensin-converting enzyme 2 . Although $80 \%$ of infections are mild or asymptomatic, patients with moderate and severe COVID-19 develop a wide range of symptoms, including respiratory, vascular and neurological complications.

Cough can persist for weeks or months after SARS-CoV-2 infection, often accompanied by chronic fatigue, cognitive impairment, dyspnoea, or pain - a collection of long-term effects referred to as the post-COVID syndrome or long COVID. A post viral cough is a distressing symptom which may be lingering for many months. Stigmatisation of patients with cough can occur, leading to social isolation, particularly during the COVID-19 pandemic. Many antitussive therapies are available, and it is yet to study a lot about the effectiveness. We currently have little understanding of the mechanisms underlying COVID-19-associated cough. This is an attempt to understand the pathophysiological mechanism of post covid cough, from available data collected from different sources and to study the scope of Homoeopathy in post covid cough.
\end{abstract}

Keywords: post covid cough, neuroinflammation, neuroimmune, neuronal hyper sensitivity, homoeopathic medicines

\section{Introduction}

The respiratory tract is the site of severe acute respiratory syndrome coronavirus 2 (SARSCoV-2) entry and infection; however, COVID-19 is a complex systemic disease, affecting the cardiovascular, renal, hematologic, and gastrointestinal and central nervous systems ${ }^{[1]}$.

Dry cough is one of the most common initial symptoms of COVID-19, reported in about 60 $70 \%$ of symptomatic patients ${ }^{[2]}$. The co-presence of cough, anosmia, and ageusia ${ }^{5}$ indicates that neuroinflammatory mechanisms might be operative in COVID-19 pathogenesis. As the cough reflex is mediated by the vagus nerve, interactions between the virus and the airway vagus nerve, with ensuing neuroinflammation, represent the likely primary events for the initiation of cough ${ }^{[3]}$.

An increasing number of reports describes an array of fluctuating or persistent symptoms experienced by patients for months after recovery from COVID-19. It is not known why the post-COVID syndrome develops in some individuals. There is emerging evidence that female sex, presence of respiratory comorbidities, and severity of acute COVID-19 presentation might be predictive of post-COVID syndrome ${ }^{[6,7]}$. So far, it is unclear whether any factors in the acute phase could specifically determine the persistence of cough. Unlike cough that persists after the common cold or flu, chronic cough in post-COVID syndrome is usually accompanied by other multisystem manifestations, which might indicate either multifactorial pathogenesis or shared mechanisms underlying these symptoms ${ }^{[3]}$.

\section{Literature review}

According to a study in The Lancet Respiratory Medicine, online surveys have found that $20-30 \%$ of people still experience a dry cough 2-3 months after becoming ill with COVID-19.

An early study from Italy found that $16 \%$ of people who recovered from COVID-19 reported still experiencing a cough 2 months after being discharged from the hospital.

More recent studies, such as the one in The Lancet Respiratory Medicine, have also found that a cough may continue for weeks to months after recovery from COVID-19. 


\section{Pathophysiology of Post covid cough explained}

Cough is a reflex that requires minimum conscious control, occurring through the activation of peripheral sensory nerves into the vagus nerves, which provide input to the brainstem at the solitary nucleus and the spinal trigeminal nucleus ${ }^{[4]}$.

It is a possibility that SARS-CoV-2 infects the sensory nerves mediating cough, leading to neuroinflammation and neuroimmune interactions as mechanisms of cough hypersensitivity ${ }^{[3]}$.

There is evidence in animals that some respiratory viruses can reach the brainstem and the brain by the retrograde route, through infection of the sensory vagal fibres from the respiratory tract. Alternatively, mechanisms might exist that trigger responses in the brain independent of intact viral particles, as shed S1 spike protein of SARS-CoV-2 can cross the blood-brain barrier in mice via absorptive transcytosis. The possible direct viral-neural interaction in the pathogenesis of cough and other sensory symptoms during SARS-CoV-2 infection is yet to be studied ${ }^{[3]}$.

Urge to cough, frequently seen in subjects with common cold and possibly also in those with acute COVID-19associated cough, has been linked to alter central processing of sensory input and cough reflex. A generalised neuronal hypersensitivity is seen in patients with post covid cough as it is associated with other symptoms like dyspnoea, chronic fatigue syndrome, and fibromyalgia ${ }^{[3]}$.

\section{Cough...how to proceed with}

Look for risk factors for long covid. If cough associated with any other cardinal symptom, it needs further clinical evaluation. If cough alone it is usually an upper airway cough syndrome. Cough with purulent expectoration indicate secondary bacterial infection. Wheeze or rhonchi indicate airway involvement. Cough with rapid shallow breathing indicates restrictive pathology.

All patients presenting with cough after covid19 may have

- Routine examination of blood

- Sputum studies for tubercular bacilli, fungal hyphae and eosinophil

- Markers of inflammation

- Procalcitonin level to rule out secondary bacterial infection

- Chest X ray

- HRCT if necessary

\section{Conclusion}

Since a generalised neuronal hyper sensitivity is the mechanism explained in persistent coughing after covid, a symptomatic approach would be better to control the same. The symptomatic presentation and the nature of cough of neuronal hypersensitivity may vary from person to person, which requires an individualistic approach.

\section{Homoeopathic Management}

The triggering factors which elicits coughing would be different. Many medicines are explained in the homoeopathic Materia medica for cough with different causative modalities.

Also we can have medicines with specific action in the air passages as the airways become more sensitive and respond very easily to common environmental allergens. Some of the medicines which can be considered in post viral cough, so also in post-covid are the following,

\section{For fatiguing irritating and exhausting cough}

Aconite, Ars alb, Arum triphyllum, Belladonna, Causticum, Corallium rubrum, Drosera, Hyosyamus, Ignatia, Ipecac, Lobelia, Mentha, Naja, Phosphorous, Scilla, Senega, Silicea, Sticta ${ }^{[8]}$.

Spasmodic, paroxysmal, nervous, suffocative cough

Aconite, Ambra grisea, Ammon brom, Arnica, Ars alb, Bell, Brom, Bryonia, Carbo veg, Caust, Chel, Cina, Conium, Corallium, Cup acet, Cup met Drosera, Hepar sulph, Hydrocyanic acid, Hyoscyamus, Ignatia, Justitia, Kali brom, Kalicarb, Kreos, Lachesis, Mercurius, Natr mur, Nitric acid Nux vomica, Osmium, Phosphorus, Rumex, Silicea, Spongea ${ }^{[8]}$.

\section{Ambra grisea}

For extreme nervous hypersensitiveness. Nervous spasmodic cough with hoarseness and eructation. Palpitation with pressure in the chest as from a lump lodged there

\section{Aralia racemosa}

Dry cough coming on after first sleep, about middle of night. Asthma on lying down at night with spasmodic cough; worse after first sleep, with tickling in throat. Constriction of chest; feels as if a foreign body were in throat. Obstruction worse in spring. Hay-fever; frequent sneezing. Rawness and burning behind sternum.

\section{Aconite}

Very sensitive to inspired air. Shortness of breath. Larynx sensitive. Stitches through chest. Cough, dry, short, hacking; worse at night and after midnight. Tingling in chest after cough.

\section{Bryonia}

Soreness in larynx and trachea. Hoarseness; worse in open air. Dry, hacking cough from irritation in upper trachea. Cough, dry, at night; must sit up; worse after eating or drinking, with vomiting, with stitches in chest, and expectoration of rust-colored sputa.

\section{Causticum}

Cough, with raw soreness of chest. Expectoration scanty; must be swallowed. Cough with pain in hip, especially left worse in evening; better, drinking cold water; worse, warmth of bed. Sore streak down trachea. Mucus under sternum, which he cannot quite reach. Pain in chest, with palpitation. Cannot lie down at night. Voice re-echoes. Own voice roars in ears and distresses.

\section{Corallium rubrum}

Throat very sensitive, especially to air. Profuse, nasal catarrh. Inspired air feels cold, suffocative cough; very rapid cough, short, barking. Cough with great sensitiveness of airpassages; feel cold on deep inspiration.

\section{Coccus cacti}

Fauces very sensitive. Suffocative cough; worse, first waking, with tough, white mucus, which strangles. Spasmodic morning cough. Walking against wind takes breath away.

\section{Eriodictyon}

A remedy for asthmatic and bronchial affections. Bronchial 
phthisis, with night-sweats and emaciation. Asthma relieved by expectoration. Cough after influenza. Furthers absorption of effusion in plural cavity. Appetite poor and impaired digestion. Whooping cough.

\section{Iodum}

Hoarse. Raw and tickling feeling provoking a dry cough. Pain in larynx. Laryngitis, with painful roughness; worse during cough. Cold extends downwards from head to throat and bronchi. Great weakness about chest. Palpitation from least exertion.

\section{Justicia}

Dry cough from sternal region all over chest. Hoarseness, larynx painful. Paroxysmal cough, with suffocative obstruction of respiration. Cough with sneezing.

\section{Kali carb}

Dry, hard cough about 3 am, with stitching pains and dryness of pharynx. Bronchitis, whole chest is very sensitive. Expectoration scanty and tenacious, but increasing in morning and after eating; aggravated right lower chest and lying on painful side.

\section{Kali iod}

Violent cough; worse in morning. Larynx feels raw. Laryngeal œdema. Awakes choking. Expectoration like soap-suds, greenish. Asthma. Dyspnœa on ascending, with pain in heart. Cold travels downward to chest.

\section{Mentha piperita}

Voice husky. Throat dry and sore, as if pin crosswise in it. Dry cough, worse from air into larynx, tobacco smoke, fog, talking; with irritation in suprasternal fossa. Trachea painful to touch.

\section{Osmium}

Acute laryngitis; cough and expectoration of tough, stringy mucus. Convulsive cough; feels as though membrane were torn from larynx. Noisy, dry, hard, cough, in violent short bursts, coming from low down, shaking the whole body. Talking causes pain in larynx. Hoarse; pain in larynx; sore sternum. Twitching of fingers, with spasmodic cough.

\section{Pix liquida}

A great cough medicine. Bronchial irritation after influenza. Constant vomiting of blackish fluid, with pain in stomach.

\section{Populus candicans}

Acute hoarseness. Throat and nostrils burn. Sits bent forward with dry cough. Pharynx and larynx feel dry, and the voice weak and toneless. Rawness and soreness of chest and throat.

\section{Rumex}

Every breath of cold air causes tickling, as from a feather or dust in throat pit and continuous cough. amel. Closing or covering the mouth or head, agg. Touching throat pit; lying on left side. Dry teasing cough preventing sleep.

\section{Sticta}

Tickling in larynx and trachea causing cough Cough: dry, < evening and night; can neither sleep nor lie down; dry, noisy; Cough after influenza; after measles; after whooping- cough;

Cough dry: Spasmodic cough which he cannot stop

\section{Spongea}

Cough dry and sibilant, sounds like a saw driven through a pine board.

\section{Reference}

1. Blomberg B, Mohn KGI, Brokstad KA et al. Long COVID in a prospective cohort of home-isolated patients. Nat 2021. https://doi.org/10.1038/s41591-021-01433-3.

2. Huang C, Wang Y, Li X, et al. Clinical features of patients infected with 2019 novel coronavirus in Wuhan, China. Lancet 2020;395:497-506.

3. Lancet Respir Med 2021;9:533-44 Published Online April 12, 2021 https://doi.org/10.1016/ S22132600(21)00125-9.

4. Canning BJ, Chang AB, Bolser DC et al. Anatomy and neurophysiology of cough: CHEST Guideline and Expert Panel report. Chest 2014;146:1633-48.

5. Menni C, Valdes AM, Freidin MB, et al. Real-time tracking of self reported symptoms to predict potential COVID-19. Nat Med 2020;26:1037-40.

6. Garrigues E, Janvier P, Kherabi Y et al. Post-discharge persistent symptoms and health-related quality of life after hospitalization for COVID-19. J Infect 2020;81:e4-6. 10.

7. Halpin SJ, McIvor C, Whyatt $\mathrm{G}$ et al. Post discharge symptoms and rehabilitation needs in survivors of COVID-19 infection: A cross sectional evaluation. J Med Virol 2021;93:1013-22.

8. Homoeopathic Materia medica William Boericke.

9. A Dictionary of practical Materia medica JH Clarke.

10. Keynotes and characteristics with comparisons $\mathrm{HC}$ ALLEN. 